\title{
CRITICAL PROPERTIES OF 2D DISORDERED 3-STATE ANTIFERROMAGNETIC POTTS MODEL ON TRIANGULAR LATTICE
}

\author{
A.K. Murtazaev ${ }^{1.2}$, A.B. Babaev ${ }^{1.3}, \underline{\text { G.Y. Ataeva }}^{1.4}$ \\ ${ }^{1}$ Institute of Physics, Dagestan Scientific Center, Russian Academy of Sciences, \\ Makhachkala, 367003 Russia \\ ${ }^{2}$ Dagestan State University, Makhachkala, 367025 Russia \\ ${ }^{3}$ Dagestan State Pedagogical University, Makhachkala, 367003 Russia \\ ${ }^{4}$ Dagestan State University of National Economy, Makhachkala, 367008 Russia \\ ataeva20102014@mail.ru
}

\begin{abstract}
By introducing a small amount of non-magnetic impurities into an antiferromagnetic (AF) two-dimensional $(2 D)$ Potts model on a triangular lattice it is that the impurities in spin systems described by this model result in the change of a first order to a second-order phase transition. The systems with linear sizes $L \times L=N, L=9-144$ are considered. Investigations are performed using the standard Metropolis algorithm along with Monte-Carlo single-cluster Wolff algorithm. On the basis of the theory of finite-size scaling, critical exponents (CE) are calculated: the heat capacity $\alpha$, the susceptibility $\gamma$, the order parameter $\beta$, and the $\mathrm{CE}$ of the correlation radius $v$.
\end{abstract}

\section{Introduction}

The Monte Carlo investigations of thermal and critical properties of magnetic systems influenced by impurities and structure defects indicate the non-ideal features of real magnetic systems to effect on phase transitions (PT) and critical properties, in particular, non-magnetic impurities can change a phase transition order [1-3]. Two-dimensional statistic systems can act as models of real physical systems, for instance, the Potts model possessing a variety in critical behavior. The most interesting object, up to date, is the triangular antiferromagnetic $2 D$ Potts model. This stems from the ability of $2 D$ lattice models to describe a large class of real physical systems: layered magnetic materials, liquid helium films, superconducting films, etc. [4].

\section{The 2Dweakly diluted antiferromagnetic Potts model}

In this work, we perform the Monte Carlo study of phase transitions and critical phenomena of impure triangular $2 D$ antiferromagnetic Potts model at a spin concentration $p=0.90$ with lattice linear sizes of $L \times L=N, L=9$-144, and periodic boundary conditions.

The Hamiltonian reads [5]

$$
H=-\frac{1}{2} J \sum_{i, j} \rho_{i} \rho_{j} \cos \theta_{\mathrm{i}, \mathrm{j}}, \quad S_{i}=1,2,3
$$

where $J$ is the parameter of nearest neighbor antiferromagnetic exchange interaction $(J<0) ; \rho_{i}=1$, if the site $i$ is occupied by a magnetic atom, $\rho_{i}=0$, if the site is occupied by a non-magnetic impurity; $\theta_{i, j}$ is the angle between interacting spins $S_{i^{-}} S_{j}$

\section{Simulation method}

To analyze a phase transition character and to define critical temperatures we use the method of fourth order Binder cumulants [6]

$$
V_{L}(T, p)=1-\frac{\left\langle E^{4}\right\rangle_{L}}{3\left\langle E^{2}\right\rangle_{L}^{2}}
$$




$$
U_{L}(T, p)=1-\frac{\left\langle m^{4}(T, p ; L)\right\rangle_{L}}{3\left\langle m^{2}(T, p ; L)\right\rangle_{L}{ }^{2}},
$$

where $E$ is the energy and $m=m_{A F^{-}}$is the order parameter of a system with $L$. Equations 2 and 3 permit an accurate evaluation of $T_{N}(p)$ at first and second order transitions, respectively. This technique works well at detection of a transition order. One knows the distinctive features inherent in PT [7]: at a first order transition the averaged value $V_{L}(T, p)$ tends to some non-trivial value $V^{*}$ according to the equation

$$
V(T, p)=V^{*}+b L^{-d},
$$

at $L \rightarrow \infty$ and $T=T_{c}(L)$, where $V^{*}$ is non $2 / 3$; the minimum value $U_{L \text {, min }}\left(T=T_{\min }, p\right)$ diverges to $U_{L, \min }\left(T=T_{\min }, p\right) \rightarrow-\infty$ at $L \rightarrow \infty$; at a second order transition the averaged value $V_{L}(T, p)$ at $T=T_{c}(L)$ will tends to $2 / 3$, and temperature dependence curves of Binder cumulants $U_{L}(T p)$ will have a well-expressed cross point. A procedure of a PT order determination was detailed in works [8-12].

\section{Results of simulation}

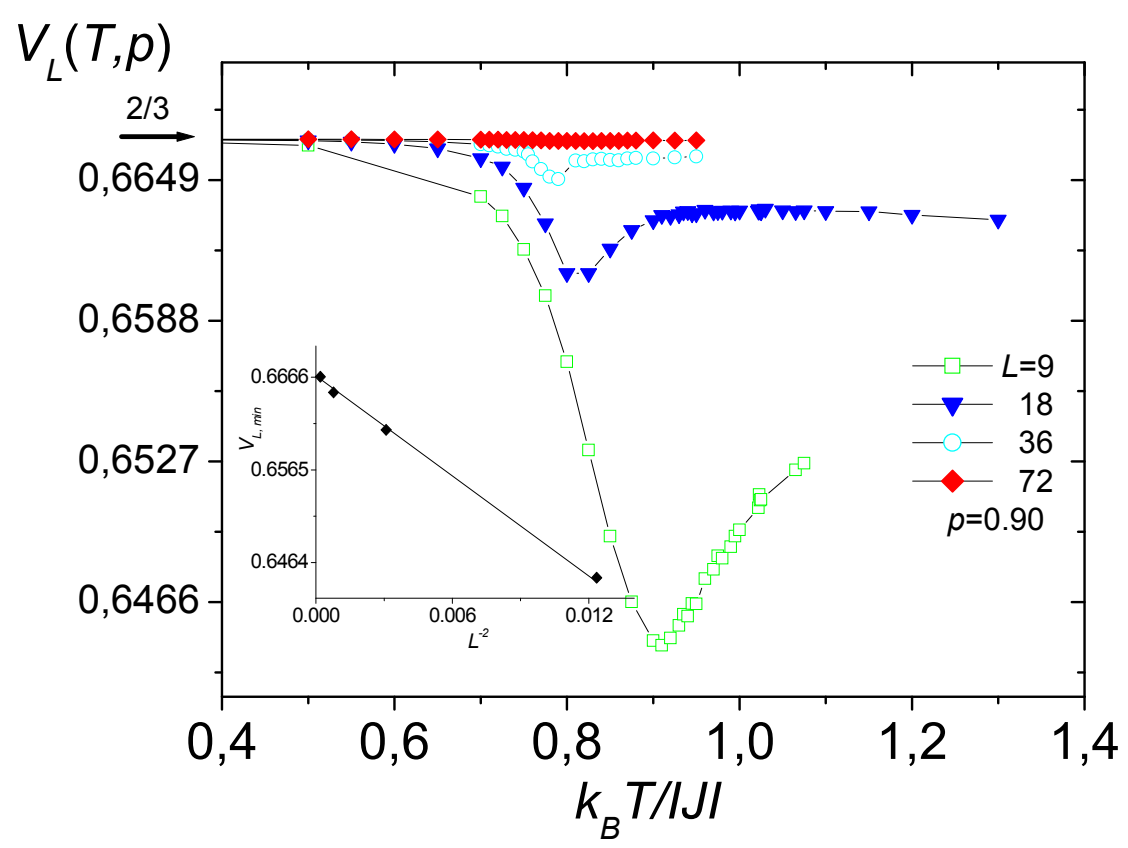

Fig. 1. The temperature dependence of Binder cumulants $V_{L}(T, p)$ for the $2 D$ 3-state sitediluted AF Potts model at $p=0.90$.

Figure1 depicts the Binder cumulants $V_{L}(T, p)$ temperature dependences for systems with different $L$ at $p=0.90$. It is apparent from Fig.1 that the $V_{L}(T, p)$ value tends to $2 / 3$ at $L \rightarrow \infty$. Such a behavior is, as has been mentioned above, typical for a second order transition [7]. Phase transition temperature $T_{l}(p)$ determined for all considered systems by the Binder cumulants method in units of $|J| k_{\mathrm{B}}$ are equal: $T_{l}(1.00)=0.940(1), T_{l}(0.90)=0.79(1), T_{l}(0.80)=0.65(2), T_{l}$ $(0.70)=0.42(3), T_{l}(0.65)=0.35(4)$.

We calculate the static CE for the heat capacity $\alpha$, susceptibility $\gamma$, and order parameter $\beta$ by means of the finite-size scaling theory (FSST). The correlations of this theory indicate that for a sufficiently large system with periodic boundary conditions the order parameter, susceptibility, and parameter $V_{n}$ for the evaluation of radius correlation ( $v$ ) critical exponents $T=T_{N}$ obey the following analytical expressions [13, 14]:

$$
\chi \sim L^{\gamma / v},
$$




$$
\begin{aligned}
& m \sim L^{-\beta / v} . \\
& V_{n}=L^{1 / v} g_{V_{n}}
\end{aligned}
$$

where $g_{V n}$ is some constant, and can act as $V_{n}$,

$$
\begin{aligned}
& V_{i}=\frac{\left\langle m^{i} E\right\rangle}{\left\langle m^{i}\right\rangle}-\langle E\rangle,(i=1,2,3), \\
& V_{3}=\frac{d U_{L}}{d \beta}=\frac{1}{\left.3 m^{2}\right\rangle^{2}}\left[\left\langle m^{4}\right\rangle E\right\rangle-2 \frac{\left.\left.m^{4}\right\rangle m^{2} E\right\rangle}{\left\langle m^{2}\right\rangle^{2}}+\left\langle m^{4} E\right\rangle,
\end{aligned}
$$

where $\beta=1 / T, T$ is the temperature. [7]

The temperature dependence of the heat capacity on $L$ is usually approximated using other expressions, for instance

$$
C_{\max }(L)=C_{\max }(L=\infty)-A L^{\alpha / v}
$$

where $A$ is some coefficient.

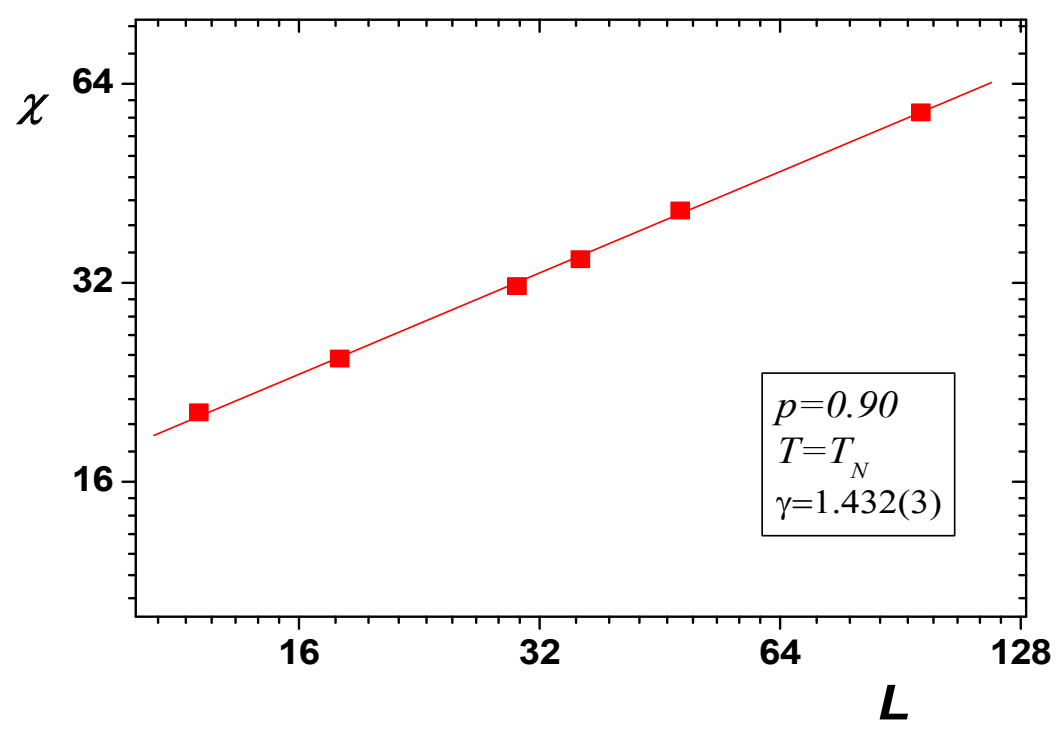

Fig. 2. The dependence of the susceptibility on $L$ at $T=T_{N}$ and $p=0.90$.

To calculate critical exponents for $\alpha, \beta, \gamma$, and $v$ we draw diagrams of the dependence of $C, m, \chi$, and $V_{n}$ on $L$. The non-linear least-square analysis allows calculation of $\alpha / v, \beta / v, \gamma / v$, and $1 / v$ values. Next, using derived $v$ values, we determine $\alpha, \beta$, $\gamma$ exponents.

In Fig. 2, we draw in log-log scale a diagram of susceptibility dependence on $L$ for the $2 D$ disordered 3 -state antiferromagnetic Potts model on triangular lattice. It should be noted that data obtained for the susceptibility don't deviate from the line even at small $L$. It is evident that the number of different initial configurations and sizes $L \geq 9$ used for averaging allow us to achieve the asymptotic critical mode. The most crucial moment is that the $v$ exponents is computed from results of the numerical experiment within the framework of this investigation, while in many other works this exponents is determined from different scaling correlations. Values of critical exponents obtained in this study are presented in Table 1 . 
Table 1.The finite-size scaling theory-based critical exponents of $2 D$ disordered 3 -state antiferromagnetic Potts model on triangular lattice.

\begin{tabular}{|c|c|c|c|c|c|c|}
\hline \multirow{4}{*}{$\begin{array}{c}\text { Our } \\
\text { data }\end{array}$} & $\begin{array}{c}\text { Spin } \\
\text { concentration }\end{array}$ & $\begin{array}{c}\text { Phase transition } \\
\text { temperature } \mathbf{T}_{\boldsymbol{l}}\end{array}$ & \multicolumn{4}{|c|}{ Critical exponents } \\
\cline { 2 - 7 } & $\boldsymbol{P}$ & $\boldsymbol{T}_{\boldsymbol{l}}$ & $\boldsymbol{\alpha}$ & $\boldsymbol{\beta}$ & $\gamma$ & 0 \\
\cline { 2 - 7 } & 1.00 & $0.940(1)$ & \multicolumn{4}{|c|}{ First order phase transition } \\
\cline { 2 - 7 } & 0.90 & $0.79(1)$ & $0.310(3)$ & $0.102(2)$ & $1.432(3)$ & $0.88(1)$ \\
\hline
\end{tabular}

\section{Conclusions}

Most valuable result of performed investigations is evaluation of the critical exponents for $2 D$ disordered 3-state antiferromagnetic Potts model on triangular lattice using the finite-size scaling theory. The obtained values of critical exponents obey the Rashbrook relation $\boldsymbol{\alpha}+\mathbf{2} \boldsymbol{\beta}+\boldsymbol{\gamma}=\mathbf{2}$. A disorder caused by non-magnetic impurities results in the change of a first order transition to a second order state. Such a behavior is associated with the suppression of the fluctuation instability caused by introduced impurities.

\section{References}

1. M. Aizenman, J. Wehr. Phys. Rev. Lett. 62, 2503 (1989).

2. K. Hui, A.N. Berker. Phys. Rev. Lett. 62, 2507 (1989).

3. A. K. Murtazaev, A. B. Babaev, G. Ya. Ataeva. J. Exp. Theor. Phys. 109 (3), 412 (2009).

4. R.J. Baxter, Exactly Solved Models in Statistical Mechanics (London: Academic Press, 1982).

5. F.Y. Wu. Rev. Mod. Phys. 54, 235 (1982).

6. K. Eichhorn, K. Binder. J. Phys.: Cond. Matter 8, 5209 (1996).

7. D. Loison, K.D. Schotte. Eur. Phys. J. B 5, 735 (1998).

8. A.K. Murtazaev, A.B. Babaev, G.Y. Aznaurova. J. Exp. Theor. Phys. 115, 6, 1042 (2012).

9. A.K. Murtazaev, A.B. Babaev. J. Magn. Magn. Mater. 324, 3870 (2012).

10. A.K.Murtazaev, A.B. Babaev, G.Ya. Aznaurova, Low Temperature physics 39, 2, 147 (2013).

11. A.K. Murtazaev, A.B. BabaevJ. Exp. Theor. Phys. 116, 1, 101 (2013).

12. A.K. Murtazaev, A.B. Babaev, JETP Lett 99,535 (2014).

13. D. Loison. Physics Letters A257, 83 (1999).

14. M.E. Fisher, M.N. Barber. Phys. Rev. Lett. 28, 1516 (1972). 